\title{
A Qualitative Study of Patient Motivation to Adhere to Combination Antiretroviral Therapy in South Africa
}

\author{
Francois van Loggerenberg, $\mathrm{PhD}^{1,2,4}$ Debra Gray, $\mathrm{PhD}^{3}$ Santhanalakshmi Gengiah, MA, ${ }^{4}$ \\ Pinky Kunene, ${ }^{4}$ Tanuja N. Gengiah, MClinPharm, MS, ${ }^{4}$ Kogieleum Naidoo, MBChB, ${ }^{4}$ \\ and Alison D. Grant, MBBS, PhD, ${ }^{2}$ for the CAPRISA 058 Study Team
}

\begin{abstract}
Taken as prescribed, that is, with high adherence, combination antiretroviral therapy (ART) has changed HIV infection and disease from being a sure predictor of death to a manageable chronic illness. Adherence, however, is difficult to achieve and maintain. The CAPRISA 058 study was conducted between 2007 and 2009 to test the efficacy of individualized motivational counselling to enhance ART adherence in South Africa. As part of the overall trial, a qualitative sub-study was conducted, including 30 individual interviews and four focus group discussions with patients in the first 9 months of ART initiation. Data were inductively analyzed, using thematic analysis, to identify themes central to ART adherence in this context. Four themes emerged that characterize the participants' experiences and high motivation to adhere to ART. Participants in this study were highly motivated to adhere, as they acknowledged that ART was 'life-giving', in the face of a large amount of morbidity and mortality. They were further supported by techniques of routine remembering, and highlighted the importance of good social support and access to supportive healthcare workers, to their continued success in negotiating their treatment. Participants in the current study told us that their adherence motivation is enhanced by free accessible care, approachable and supportive healthcare workers, broad social acceptance of ART, and past first-hand experiences with AIDS-related co-morbidity and mortality. Programs that include specific attention to these aspects of care will likely be successful in the long term.
\end{abstract}

\section{Introduction}

\section{Adherence to antiretroviral therapy}

$\mathbf{T}$ HE SUCCESS AND IMPACT of antiretroviral therapy (ART) has transformed human immunodeficiency virus (HIV) infection and disease from a terminal illness to a manageable chronic illness. ${ }^{1,2}$ Taken as prescribed, that is with high levels of treatment adherence, combination ART leads to improved immune functioning, with a decreased risk of progression to AIDS and death. ${ }^{3}$ ART is not only important for physical wellbeing, but has been associated with an increased probability of being at work within 6 months of starting therapy in Uganda, and elsewhere in Africa. ${ }^{4}$
The key to effective ART treatment is patient adherence and retention in care. Patient adherence is the percentage of pills taken correctly as prescribed over a defined time period. It has been estimated that for HIV treatment to be effective adherence of at least $95 \%$ is required. ${ }^{5}$ While it is debatable whether this very high level of adherence is necessary for all ART drug combinations, viral suppression has been seen in lower levels of adherence for the more potent non-nucleoside reverse-transcriptase inhibitor-based therapy or in boosted protease inhibitor therapy, ${ }^{6,7}$ it is nonetheless accepted that for ART to work the highest level of adherence possible is a desirable goal. Inadequate levels of adherence may lead to the development of drug resistance and treatment failure,

\footnotetext{
${ }^{1}$ The Global Health Network, Centre for Tropical Medicine and Global Health, University of Oxford, Oxford, United Kingdom.

${ }^{2}$ London School of Hygiene and Tropical Medicine, London, United Kingdom.

${ }^{3}$ University of Winchester, Winchester, United Kingdom.

${ }^{4}$ CAPRISA, University of KwaZulu-Natal, Durban, South Africa.
}

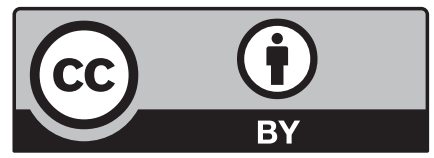

(c) van Loggerenberg 2015; Published by Mary Ann Liebert, Inc. This Open Access article is distributed under the terms of the Creative Commons License (http://creativecommons.org/licenses/by/4.0), which permits unrestricted use, distribution, and reproduction in any medium, provided the original work is properly credited. 
with very serious consequences for the individual and the treatment programme as a whole. ${ }^{8-11}$

Studies that focus on adherence to ART in Africa, and other resource-constrained countries, suggest that good adherence depends on timely and frequent clinic attendances and retention in care programmes, which present particular challenges to patients in these settings. ${ }^{12}$ Retention within treatment brings with it the cost of transport to clinics, time away from essential functions such as work, and user costs and fees that for patients may be unsustainably high. ${ }^{13-16}$ Studies in Uganda, Burkina Faso, and Mali demonstrated that distance to the clinic, and related high transport costs, are risk factors for insufficient access to care and inadequate viral suppression ( $\geq 500$ copies/ $\mathrm{mL}$ after at least 6 months of treatment). ${ }^{17,18}$ Additionally, a key review of ART adherence in Africa and Brazil highlighted that self-funded ART is associated with poor adherence, as either inadequate regimens are purchased, or prescriptions are filled erratically. ${ }^{19}$ This review also found that in countries where costs fell, adherence tended to improve.

Alongside the costs involved, factors that further impact ART adherence are alcohol abuse, lack of adequate counselling, long waiting times for results, and poor provider-patient relationships. ${ }^{20,21}$ Fear of stigma, especially from sexual partners, and low rates of disclosure were significant in South African studies. ${ }^{22}$ Being able to collect, store, and correctly take HIV medicine consistently over time in secret and without social and material support is likely to be very difficult, and so disclosure to at least one other trusted person is seen to be a very important component of good adherence and is the foundation of the treatment supporter approach to ART. ${ }^{23}$ Being able to disclose to someone who does not react negatively can reduce perceived stigma, and feeling less stigmatised may mean that individuals are more likely to disclose their status voluntarily, in order to receive much needed support and to improve adherence. ${ }^{24,25}$ In this sense then, disclosure to at least one other supportive person, who is able to provide material and/or social support, is seen as important, and full disclosure may not be necessary to improve treatment adherence, and may even be undesirable where this may lead to increased stigma and discrimination from unsupportive people. ${ }^{26}$

In South Africa, higher rates of disclosure tended to be associated with good adherence. However, such disclosure was often difficult for patients to negotiate. For example, if participants feared being stigmatized by their sexual partner, then they are significantly less likely to disclose their HIV status and report that they were adherent. Moreover, research from the Western Cape province of South Africa found that alcohol abuse in family members is often a key limiter to disclosure, in that it was difficult to trust someone who used alcohol to be discreet. ${ }^{23}$

Other qualitative studies from Africa have suggested that higher levels of adherence may be related to the motivation to be able to provide for families under difficult conditions. ${ }^{14}$ In some African cultures, on-going treatment in the face of resolved symptoms may seem foreign. ${ }^{15}$ Finally, concerns about food security, and being able to support improved appetites from successful treatment, have also been raised as barriers to treatment adherence. ${ }^{16}$

\section{The Current Study}

Data for this qualitative study were collected as part of a randomized control trial (RCT), CAPRISA 058, which aimed to test the efficacy of an individualized motivational counseling intervention to enhance ART adherence. ${ }^{27} \mathrm{We}$ conducted this RCT in response to concerns that, due a lack of political support for HIV treatment and, more damagingly, explicit support of nutritional supplements, vitamins, and other means of 'treating' HIV, ${ }^{28-30}$ patients may believe that that ART was no better than these 'treatments' or even poisonous. ${ }^{31}$ The majority of our participants were also enrolled on a programme to integrate HIV and tuberculosis (TB) treatment, since active TB is often a common entry point for HIV testing and ART initiation. ${ }^{32}$ We were concerned that the increased pill burden, the potential for greater side effects, and the fact that they have different treatment regimes, could all negatively impact on HIV treatment adherence.

In response to these concerns, an enhanced adherence support intervention was developed (described in the parent study ${ }^{27}$ ). The primary study showed that the participants who received enhanced adherence support had no greater virological suppression at 9 months than the control arm participants $(87.9 \%$ vs. $89.8 \%, p=0.62$ ). Additionally, there was no difference in the proportion of people in each arm who achieved $>95 \%$ adherence by pill count at 6 months $(79.5 \%$ vs. $82.9 \%, p=0.51)$. Importantly, all patients-including those in the control arm who received standard adherence support-achieved unexpectedly good levels of adherence and treatment outcomes. In this article, we explore how and why these patients had managed to maintain such high levels of adherence, through an analysis of qualitative data to provide insight into their motivations, strategies, and experiences of ART, as well as to evaluate the validity of the quantitative results in this context. ${ }^{33}$

\section{Methods}

\section{Design}

The data for this qualitative study were collected through interviews and focus groups with patients scheduled for their 9-month post-treatment initiation clinic visit in both study arms. Participants not selected for interviews but willing to take part in focus group discussions were invited to do so, during this same period. Data were collected by a staff member working on HIV prevention studies at the clinic, but not on the current study or on the treatment programme. This was done in order to promote more open and honest reflection by patients of their treatment at the clinic as, if someone working on the treatment programme collected the data, participants might feel pressure to present their treatment experiences and adherence behavior in favorable light.

The interviews were semi-structured, consisting of a series of broad open-ended questions, which the interviewer was encouraged to prompt. ${ }^{34}$ These included questions about personal experience of taking ART, personal adherence strategies, examples of non-adherence, experiences of the level of care at the clinic, side effect experiences, experiences and attitudes towards disclosure, and other community resources relating to adherence support. A final section of the guide dealt with questions relating to more private behaviors, such as drug and alcohol use, as well as sexual behavior. The language was permissive to encourage discussion around non-adherence. Talking about unanticipated issues was encouraged, and the interviewer was trained to facilitate this.

The four focus groups were conducted to encourage interaction more akin to everyday (and more social) conversation, 
and they have also been found to allow participants to express views that are more critical than those presented in individual interviews. ${ }^{35}$ This was seen to be especially useful in assessing experiences at the treatment clinic, as views critical of the care provided at the clinic would be important to assessing the value of the adherence support.

\section{Participants}

30 patients consented to take part in individual interviews, 14 with control arm participants and 16 with intervention arm participants. The interviewees consisted of 13 males (mean age 39.5 years, SD 7.3) and 17 females (mean age 34.8 years, SD 6.3). Six of the men and eight of the women were in the control arm. Four focus groups were conducted with four participants in each focus group. The composition of the groups depended on which participants were available on the day. Three of the four focus groups consisted of intervention arm participants; one was all male, one all female, and one mixed. The group consisting of control arm participants was of mixed gender.

\section{Data collection and analysis}

All interviews and focus groups were conducted in isiZulu, the language of preference for all participants, and were digitally recorded, transcribed, and translated into English. The English transcripts constituted the study data.

Data were analyzed in NVivo ${ }^{\mathrm{TM}}$ 8.0, using thematic analysis, which is a methodology aimed at capturing patterns in data, guided by six phases of coding. ${ }^{36}$ Transcripts were coded by two of the authors (Francois van Loggerenberg and Debra Gray) independently and, at the time of coding, the allocation to intervention or control arm was hidden, as was any other participant demographic information. First, the transcripts were read, and re-read for evidence of adherence strategies and experiences regarding adherence, and initial codes were noted. The codes were then organized into potential themes, which were then reviewed to see if these themes were relevant to the whole dataset. Emerging themes were identified as being 'key' if they were common across the sources, as well as if important information in relation to adherence to ART was discussed by participants, even if this was not prevalent in the dataset. So, for example, negative experiences with adherence were very uncommon but considered key as they were very informative about the overall lack of issues experienced by participants. Finally, the two independent analyses of the data were combined and compared. There was a high level of consistency between the two analysts, and the four themes that emerged most clearly from both analyses are presented below.

\section{Results}

Given that no differences in adherence and viral load outcomes were found between the two arms in the primary study trial, the findings presented below do not compare participants' experiences of these different forms of counseling. Rather, we concentrate on the ways in which participants across both counseling groups reported and negotiated the various challenges and issues related to taking and adhering to ART. We focus here on four themes that relate to important aspects of these participants' experiences of ART adherence: 'ART is for Life,' 'Routine Remembering,' 'Support and Disclosure,' and 'Good Clinics, Good Carers.'

\section{$A R T$ is 'for life'}

Across the data, many participants spoke of a strong personal motivation to adhere to their treatment regime, and it was clear that this motivation was linked to the idea that ART was 'for life' - that is, the medication was described by participants as 'life-giving' and, therefore, as a 'life-long' commitment. Consider the following extracts:

Extract 1 (Focus Group 4, Control Arm, Participant 4):

I have noticed how beautiful I have become after taking these tablets-I don't think I will ever stop taking them. I was sick, I was very sick, I'm not exaggerating...I was raised from the dead.

Extract 2 (Focus Group 1, Intervention Arm, Participant 4):

You can't stop taking these tablets because...like me, they woke me up from the grave. They are now my life. I will only stop taking them when I'm dead, when I'm no more, because they are my life.

These extracts demonstrate the ways in which, for these participants, adhering to ART is understood to be a 'life-ordeath' matter; an understanding that in turn exerts very strong motivational push on participants to remain on their medication. As in Extract 1, this link between ART and 'life' was typically attributed to the transformative effects of the medication-from a state of being 'sick' to one of health-and many participants spoke about how taking the medication has effected very real bodily transformations (e.g., on eating, sleeping, tiredness, and appearance) that are taken as evidence for the life-giving force of ART.

For many participants, even the side-effects of ART's (such as diarrhea, nausea, and transient dizziness), which have been documented elsewhere as barriers to adherence, were seen as manageable relative to the ill-health experienced prior to starting ART. ${ }^{37,38}$ For example, when asked whether she had ever thought of not taking her medication one participant noted: 'I once thought of that when I started taking them, when I was still experiencing side effects but I asked myself if I stop them, how am I going to live (Interview 7, Female, 36 years, Control Arm).

While the notion that 'ART is for life' did seem to successfully motivate adherence behavior in these participants, it also seems that the life-long commitment of ART can act as a barrier to starting treatment in the first place. For example, the following participant is discussing how she didn't take her tablets for the first couple of days after she was first given them:

Extract 3 (Focus Group 1, Intervention Arm, Participant 2): There was a time when I didn't drink them. I didn't take them on the first day that I was supposed to take them. They asked me if I was going to take them, I said, "yes I was going to take them." I took them and put them aside, I didn't drink them. What came to my mind at that time was that they say once I start taking ARVs, I would have to take them for the rest of my life. I asked myself "until when would I take these tablets?" I was scared.

For some participants, this fear is clearly linked to a variety of socially produced and widely available myths about ART in the communities in which they live, for example, the idea that ART results in poor health, makes you die faster, and that it is stopping the tablets that make you die (rather than the HIV)_reinforced by the metabolic complications of treatment. ${ }^{39}$ Such social myths may constitute a potential barrier 
to others coming forward, being tested and starting treatment. ${ }^{40}$ It may be that these misunderstandings of ART may constitute a barrier to correct ART adherence in populations with less access to counseling and other forms of support.

\section{Routine remembering}

Forgetting to take pills is often cited as a reason for nonadherence. Indeed, in unpublished quantitative data from the primary study, nearly $38 \%$ of participants (34/90) who acknowledged missing doses indicated that this was due to simple forgetting. However, in these qualitative data from the study, very few participants talked about having any problems with remembering to take their pills. Instead, participants spoke of a variety of practical strategies that allowed them to remember to take their medication in a highly routine way-often using multiple strategies simultaneously.

By far the most popular reminder related to cell phone or clock alarms which alone accounted for half of the around 40 individual strategies mentioned. Having someone remind them to take their pills accounted for a quarter of these strategies. Linking pill taking to popular TV programmes (such as popular local soap operas) was also noted by some participants, whilst others linked their pill taking to other daily routines (e.g., taking the pills directly after waking). The follow extract is typical of the way in which participants describe their efforts to remember to take their medication, including the ease with which they describe this being done:

Extract 4 (Interview 24, Female, 47 years, Control Arm): I make sure that I eat my supper at seven, then at nine I take my tablets. And I also make sure that I set alarm on my phone to make sure that it reminds me at this time to take my tablets. Also this box I received from the clinic where I put my tablets, it also reminds me of the days, I make sure that I don't miss any day without taking my tablets.

For many, this process was made easier by the fact that they had been on TB medication prior to starting ART. For example, this participant who previously had to be on TB treatment typifies how TB treatment experience may support ART adherence:

Extract 5 (Interview 8, Male, 39 years, Intervention Arm): I had no fears about ARVs because I'm used to taking tablets. I have taken too many tablets in my life. And the tablets I am taking now are far less because I only take four tablets.

In general, participants described adherence as being a process of habituation-as something that they had to get used to and have to 'work with' before arriving on a successful routine. For example, some participants did report that they had problems remembering to take their medication exactly on time when they first started-a problem that was often resolved by changing their medication routine (e.g., the time that they took it) or their daily routine (e.g., the time they went to bed) to something that worked for them. Clearly, the counter to this highly routine and habitual form of remembering is that any deviation from a normal daily routine will clearly lend itself to forgetting. However, these participants appeared to successfully negotiate and cope with this possibility with relative ease. For example, as one participant reports: 'It's in my system now, I know exactly time to take my treatment (Focus group 4, Participant 3).

\section{Support and disclosure}

Across the data sources, many participants described how it was important for them, and, others to disclose their HIV status. Disclosure was not quantitatively assessed in this study, and it cannot be presumed that the majority of participants in the study had disclosed, or that self-reported disclosure reflected actual disclosure rates. However, it was clear that most participants did see disclosure as an important facilitator for adherence-both directly in the sense that other people could remind participant to take their medicine (as in Extract 4 above), and indirectly in the sense that other people were seen as key to reducing the stress on treatment patients. For example:

Extract 6 (Focus Group 2, Participant 2):

It is important to have at least one person who knows so that she can remind you. Even if you don't live together, she can even buzz you on the phone to remind you that it's about time to take your medicines. And also it helps to release stress because you don't have to carry the burden that is eating only you inside.

However, participants still reported some concerns about disclosing their HIV status to others. This is unsurprising given that these participants live within communities and contexts where stigma and discrimination against HIV positive individuals is high. ${ }^{26,41}$ Indeed, several participants expressed concern that being on ART was still associated with immoral behavior in communities and that they were afraid of being victimized, or being ostracized from their communities. Others indicated that they did want to be blamed as the person who brought HIV to a relationship. In part, these issues are related to the extent of gender-based violence associated with HIV infection in southern Africa, which is clearly a barrier to disclosure..$^{25,42,43}$

Generally, participants recognized that non-disclosure made it difficult to adhere to treatment regimes, for example, through having to hide pill-taking, missing clinic appointments, or not being able to discuss condom use to prevent reinfection, which was seen as key to continued health. Some participants also talked about how they found that it was easier to hide their HIV treatment if they had previously disclosed their TB status. For example, one participant talked about how they were able to avoid using alcohol by referring to how sick they were with TB, and another participant was able to use her TB treatment to ensure that her partner used condoms, even though he was not yet aware of her HIV status.

It is important to note that, while many participants did express these wider concerns about disclosure, some participants viewed disclosure as a positive community action. Particularly that disclosure to the right people at the right time was a strategy for ensuring that individuals accessed treatment when needed. Indeed, many participants had stories to tell about how their own openness had positive consequences for getting other people onto treatment. Participants who have done well on ART may feel a responsibility to the community to ensure that the misconceptions about treatment are dispelled and that they are examples of how well treatment can work.

Finally, while few interviewees felt that disclosure was not desirable, there were some exceptions to this, and their views are informative. The following participant related a negative 
outcome to testing and disclosure, which motivated current non-disclosure.

Extract 7 (Interview 14, Male, 41 years, Control Arm): I told myself that, since both myself and her came together for test at this clinic, I thought she was going to accept it but in the end she couldn't accept it. I was scared of people in such a way that most people don't even know that I have this disease. I kept it to myself and I'm taking care of myself. I also make sure that I take my tablets because I don't want many people to know because if I get sick, they will end up knowing because doctors will report that it's because I'm not taking the treatment correctly, hence I'm getting sick.

Ironically, the fear of appearing ill and therefore being known to be sick was motivating for this participant to adhere to treatment. The motivational or adherence-enhancing potential of fear of disclosure would need to be weighed against the significant difficulties associated with taking medication correctly, but covertly.

\section{Good clinic, good carers}

All of the participants talked about the quality of care they receive at the research clinic and how important this was to their treatment, adherence, and general well-being. It is clear that this may (at least in part) be because these participants felt some pressure to be positive about the programme in an interview or focus group format. However, what is perhaps most interesting about these accounts is not their endorsement of the clinic, but the kinds of things that they saw as being helpful to their continued adherence and health. For example, several patients indicated that it was important that the research clinic was a place of (nonjudgmental) empathy and support. The fact that this facility is a dedicated HIV treatment clinic meant that participants felt comfortable being together in the waiting room. They also felt that staff had been well trained to be sensitive to HIV patients. Similarly, several patients noted that the clinic staff had helped them to understand their medication and treatment regimes, thereby making it easier for them to understand what they had to do and why. Nearly all of the participants also commented on the range of additional services that the research clinic provided, for example, tracking and tracing by phone call, delivering pills to their home, or providing a taxi service if they needed to come to the clinic-all of which were seen as essential to their continued treatment success.

Across the focus groups and interviews, it was clear that patients trusted the clinic staff. This is in turn meant that they were able to go to the staff with issues and concerns. For example, when asked about side effects a majority of patients mentioned that they either had or would tell the nurse or doctor about them, which meant they could be resolved quickly and relatively easily. For example:

Extract 8 (Interview 7, Female, 36 years, Control Arm):

Yeah, they troubled me somehow, in a way that they drugged me, at night I will have bad dreams, you know such things. But I decided to continue because they had explained to me that the treatment would do that initially but it will go away. So I didn't decide to stop them just because I was experiencing all those things, but instead I continued taking them.

Similarly, the two participants in the study who did indicate that they had any problem with adherence reported that they returned to the research clinic to tell the clinic staff, at which point they received additional counseling about the importance of adherence from both the doctor and the counselor.

For many participants, the high levels of care described above were directly contrasted with the perceived and experienced standard of care found in public sector clinics in South Africa. One participant described this process in terms that are actually very damaging, and illegal:

\begin{abstract}
Extract 9 (Focus group 2, Intervention Arm, Participant 1): I would say it is very good and respected because they know our status. We don't have a problem with them and we are not scared of them. In other places they shout at you in front of everyone. Sometimes people wake up in the early hours of the morning and they have to pay the nurse so that she can give them their files to take the front row. If you don't pay even if you come in the morning, you can sit on the queue forever. I accompanied my friend and we arrived in the morning, we were told that those who come late have to pay to get their files and if you pay then they put your file on top and the other at the bottom. So there is no respect at all. They have an attitude towards you as if you deliberately choose to be HIV positive.
\end{abstract}

For our participants, there was a general perception that patients at other clinics did not do as well (and at times died) due to the care received, even where they were on ART. Additionally, some participants felt that the medication used in the study clinic was more effective than treatments given elsewhere. This idea may have arisen because the pills given at the study clinic were once-a-day regimens, tailored to be compatible with TB treatment, and not exactly the same regimen as that given at the public health clinics.

\section{Discussion}

Given the expected complexity of ART in the context of high levels of TB treatment, the good treatment and adherence outcomes found in this study were unexpected. Our data suggest that this is primarily because the participants had very strong 'life or death' motivations to adhere, had developed highly routinized ways of remembering, relied on disclosure to key others, and relied on the clinic staff and procedures to help them to remember to take their pills and take them correctly.

The 'life or death' motivation of these patients may well be because participants had experienced the deaths of others, or had experienced what it is like to be very ill and not have access to treatment. Therefore these participants valued the treatment more than in other contexts where access may be easier, and options more prolific. With such high levels of expressed motivation, it is not surprising that efforts to improve motivation in the intervention arm were unable to lead to greater levels of treatment success in that arm. The physical transformation from 'sick' to 'well' (or from 'death' to 'life') is an important aspect in continued adherence in our participants, and has been found to be a key component of reestablishing social support networks in other populations, as in Nigeria, for example. ${ }^{44}$

Participants report that TB treatment was a useful way to access HIV diagnosis and treatment, which is an important contribution of this study. For many participants, being adherent to HIV treatment was seen as being easier because they had already had some experience of routine pill taking and were therefore less likely to forget to take their pills, or because 
it helped them to hide their HIV treatment from others. Thus, it would seem that treatment for TB-which may be less stigmatized and stigmatizing than treatment for HIV - can provide patients with ways of being covertly adherent.

It is clear that the adherence support provided to patients by the clinic is key to their retention in care and, therefore, adherence success, and this is amenable to improvement in clinics where participants are not managing to adhere to their treatment. On the one hand, this relates to the counseling support, which was clearly successful in engendering highly routinized remembering, as well as providing key skills that enabled patients to overcome specific issues relating to nonadherence (e.g., side-effects, missed clinic visits, alcohol use). This also relates to the various forms of practical support provided. The research clinic offered very efficient tracking and tracing procedures, and was also very centrally situated, with easy access to key public transport routes. Thus, participants are likely to have experienced fewer transportation issues than might have been the case if the clinic had been in a more remote area. ${ }^{17,18}$ Some compensation was also paid to those participants enrolled in research studies at the site, to assist with transport and for the extra time spent at the clinic. This is very different to experiences in the public sector, where patients may be asked to pay a nominal amount for treatment; something which may be further compounded by the need to pay bribes (e.g., to receive preferential queuing, if these reports are substantiated).

Overall, it is highly likely that these various forms of support were central to promoting consistent clinic attendance and that retention in care as essential for continued high adherence. It is well documented that where participants have difficulty accessing medical care and services, they are unlikely to be able to adhere to their medication and care visits. ${ }^{17}$ Qualitative data from Nigeria, Tanzania, and Uganda also suggest that missed visits, most often for unintentional reasons, may lead to reluctance to return to the clinic, which in turn leads to long-term disengagement with care. ${ }^{45}$ Similarly, other studies in Africa and India have found that the financial costs of treatment can impact, usually negatively, on adherence. ${ }^{19,46,47}$ This means that the results of this study may not be easily generalized to all clinics in South Africa (or indeed other resource constrained settings), as the level of support offered is not comparable to, for example, clinics in more remote areas where transportation is a key issue.

A key strength of this study is that we used data from one of a first wave of adherence RCTs in Africa, and did not rely purely on self-reported measures of adherence, rather it included viral load as the primary outcome, which in this context was likely to be the best indicator of good treatment adherence. In addition to the quantitative outcomes, this study further improved the relevance of findings by including a substantial qualitative study component, which has been outlined here. These data provide a great deal of insight into the outcomes of the RCT, information that would otherwise be lacking in the standard quantitative approach, and show the strength of a mixed-methods approach to understanding behavioral interventions. ${ }^{33}$ Where the quantitative study report outlines 'what' happened, this qualitative study is able to fill in more of the 'why', from the lived experience of the participants. The high levels of motivation and lack of serious problems expressed in the qualitative data support and validate the quantitative study outcomes.
The interviewees also were, on the whole, very positive about their experiences at the clinic, which could also be due the fact that within the healthcare system in South Africa, access to ART was still problematic, and participants may have been reticent to speak about any issues with ART provision as they may have feared losing their access to treatment. It should be noted that these data were collected at 9 months post-initiation of ART, and treatment fatigue may yet set in, but longer follow up was beyond the scope of this study.

Participants in the study told us that their adherence motivation is enhanced by free accessible care, approachable and supportive healthcare workers, broad social acceptance of ART, and past first-hand experiences with AIDS related co-morbidity and mortality. Of these factors, the approachable and supportive healthcare environment is the one that is most amenable to improvement to enhance the likelihood that participants will be retained in care, and thus benefit the most from the positive health outcomes that come from sustained high adherence. Programs that include specific attention these aspects of care will likely be successful in the long term.

The strong life or death motivation expressed by the participants is clearly important in ensuring that participants continued to take their treatment as prescribed, and at sufficient levels of adherence in face of key personal challenges. The danger being that as patients become more 'well' the impact of these motivations may fade and long-term adherence may be poorer as a result, which is something that would need to be monitored in any successful treatment programme. The negative and damaging perceptions of the public health sector facilities are concerning, and should be explored further.

Finally, the finding that our participants found experience with TB treatment facilitated entry and retention on the ART treatment program, was unexpected but very reassuring for the continued success of integrating TB and HIV treatment in a setting like South Africa where both disease, and co-infection, are very prevalent. This qualitative data is supportive of quantitative data that indicates that integrated treatment is preferable in this setting. ${ }^{48,49}$

\section{Acknowledgments}

We would like to thank the clinic research support staff, who provided the data collection, nursing services, as well as the administrative and counseling support for CAPRISA 058 study, Senzo Hlathi, Goodness Gumede, and Thandi Shezi. Dr. Leila Mansoor provided advice and assisted with the training of the clinic counseling staff. Drs. Katherine Fielding and Simon Lewin provided support as members of the PhD advisory committee. We would like to especially acknowledge the participants who took part in this study.

\section{Author Disclosure Statement}

No competing financial interests exist.

\section{References}

1. Broder $\mathrm{S}$. The development of antiretroviral therapy and its impact on the HIV-1/AIDS pandemic. Antiviral Res 2010;85:1-18.

2. Jones R, Gazzard B. The cost of antiretroviral drugs and influence on prescribing policies. Int $\mathrm{J}$ STD AIDS 2006;17:499-506. 
3. Amico KR, Harman JJ, Johnson BT. Efficacy of antiretroviral therapy adherence interventions: A research synthesis of trials, 1996 to 2004. J Acquir Immune Defic Syndr 2006;41:285-297.

4. Linnemayr S, Glick P, Kityo C, Mugyeni P, Wagner G. Prospective cohort study of the impact of antiretroviral therapy on employment outcomes among HIV clients in Uganda. AIDS Patient Care STDS 2013;27:707-714.

5. Paterson DL, Swindells S, Mohr J, et al. Adherence to protease inhibitor therapy and outcomes in patients with HIV infection. Ann Intern Med 2000;133:21-30.

6. Bangsberg DR. Less than $95 \%$ adherence to nonnucleoside reverse-transcriptase inhibitor therapy can lead to viral suppression. Clin Infect Dis 2006;43:939-941.

7. Shuter J, Sarlo JA, Kanmaz TJ, Rode RA, Zingman BS. HIV-infected patients receiving lopinavir/ritonavir-based antiretroviral therapy achieve high rates of virologic suppression despite adherence rates less than 95\%. J Acquir Immune Defic Syndr 2007;45:4-8.

8. Ballif M, Ledergerber B, Battegay M, et al. Impact of previous virological treatment failures and adherence on the outcome of antiretroviral therapy in 2007. PLoS One 2009; 4:e8275.

9. Lima VD, Harrigan R, Bangsberg DR, et al. The combined effect of modern highly active antiretroviral therapy regimens and adherence on mortality over time. J Acquir Immune Defic Syndr 2009;50:529-536.

10. Lima VD, Harrigan R, Murray M, et al. Differential impact of adherence on long-term treatment response among naive HIV-infected individuals. AIDS 2008;22:2371-2380.

11. Lima VD, Johnston K, Hogg RS, et al. Expanded access to highly active antiretroviral therapy: A potentially powerful strategy to curb the growth of the HIV epidemic. J Infect Dis 2008;198:59-67.

12. Hardon A, Davey S, Gerrits T, et al., eds. From access to adherence: The challenges of antiretroviral treatment: Studies from Botswana, Tanzania and Uganda 2006. Geneva: The World Health Organization, 2006.

13. Castro A. Adherence to antiretroviral therapy: Merging the clinical and social course of AIDS. PloS Med 2005;2:e338.

14. Crane JT, Kawuma A, Oyugi JH, et al. The price of adherence: Qualitative findings from HIV positive individuals purchasing fixed-dose combination generic HIV antiretroviral therapy in Kampala, Uganda. AIDS Behav 2006;10:437-442.

15. Murray LK, Semrau K, McCurley E, et al. Barriers to acceptance and adherence of antiretroviral therapy in urban Zambian women: A qualitative study. AIDS Care 2009;21: 78-86.

16. Weiser SD, Tuller DM, Frongillo EA, Senkungu J, Mukiibi $\mathrm{N}$, Bangsberg DR. Food insecurity as a barrier to sustained antiretroviral therapy adherence in Uganda. PLoS One 2010;5:e10340.

17. Boileau C, Nguyen VK, Sylla M, et al. Low prevalence of detectable HIV plasma viremia in patients treated with antiretroviral therapy in Burkina Faso and Mali. J Acquir Immune Defic Syndr 2008;48:476-484.

18. Tuller DM, Bangsberg DR, Senkungu J, Ware NC, Emenyonu N, Weiser SD. Transportation costs impede sustained adherence and access to HAART in a clinic population in Southwestern Uganda: A qualitative study. AIDS Behav 2010;14:778-784.

19. Orrell C. Antiretroviral adherence in a resource-poor setting. Curr HIV/AIDS Rep 2005;2:171-176.
20. Duff P, Kipp W, Wild TC, Rubaale T, Okech-Ojony J. Barriers to accessing highly active antiretroviral therapy by HIV-positive women attending an antenatal clinic in a regional hospital in western Uganda. J Int AIDS Soc 2010;13:37.

21. Mills EJ, Nachega JB, Bangsberg DR, et al. Adherence to HAART: A systematic review of developed and developing nation patient-reported barriers and facilitators. PLoS Med 2006;3:e438.

22. Nachega JB, Stein DM, Lehman DA, et al. Adherence to antiretroviral therapy in HIV-infected adults in Soweto, South Africa. AIDS Res Hum Retroviruses 2004;20:10531056.

23. Nachega JB, Knowlton AR, Deluca A, et al. Treatment supporter to improve adherence to antiretroviral therapy in HIV-infected South African adults: A qualitative study. J Acquir Immune Defic Syndr 2006;43:S127-S133.

24. Lyimo RA, Stutterheim SE, Hospers HJ, de Glee T, van der Ven A, de Bruin M. Stigma, disclosure, coping, and medication adherence among people living with HIV/AIDS in Northern Tanzania. AIDS Patient Care STDS 2014;28:98105 .

25. Dlamini PS, Wantland D, Makoae LN, et al. HIV stigma and missed medications in HIV-positive people in five African countries. AIDS Patient Care STDS 2009;23:377387.

26. Dlamini PS, Kohi TW, Uys LR, et al. Verbal and physical abuse and neglect as manifestations of HIV/AIDS stigma in five African countries. Public Health Nurs 2007;24:389-399.

27. van Loggerenberg F, Grant $A D$, Naidoo K, et al. Individualised motivational counselling to enhance adherence to antiretroviral therapy is not superior to didactic counselling in South African patients: Findings of the CAPRISA 058 Randomised Controlled Trial. AIDS Behav 2015;19: 145-156.

28. Nattrass N. South Africa's "Rollout" of highly active antiretroviral therapy: A critical assessment. J Acquir Immune Defic Syndr 2006;43:618-623.

29. Ojikutu B, Makadzange AT, Gaolathe T. Scaling up ART treatment capacity: Lessons learned from South Africa, Zimbabwe, and Botswana. Curr HIV/AIDS Rep 2008;5: 94-98.

30. Chigwedere P, Seage GR, 3rd, Gruskin S, Lee TH. Estimating the lost benefits of antiretroviral drug use in South Africa. J Acquir Immune Defic Syndr 2008;49:410-415.

31. Mitchell SK, Kelly KJ, Potgieter FE, Moon MW. Assessing social preparedness for antiretroviral therapy in a generalized AIDS epidemic: A diffusion of innovations approach. AIDS Behav 2009;13:76-84.

32. Abdool Karim SS, Abdool Karim Q, Friedland G, Lalloo U, El-Sadr WM. Implementing antiretroviral therapy in resource-constrained settings: opportunities and challenges in integrating HIV and tuberculosis care. AIDS 2004;18:975979.

33. Sankar A, Golin C, Simoni JM, Luborsky M, Pearson C. How qualitative methods contribute to understanding combination antiretroviral therapy adherence. J Acquir Immune Defic Syndr 2006;43:S54-S68.

34. Britten N. Qualitative interviews. In: Pope C, Mays N, eds. Qualitative Research in Health Care. 3rd ed. Malden, MA: Blackwell Publishing Ltd, 2006, pp. 12-20.

35. Kitzinger J. Focus groups. In: Pope C, Mays N, eds. Qualitative Research in Health Care. 3rd ed. Malden, MA: Blackwell Publishing Ltd, 2006, pp. 21-31. 
36. Braun V, Clarke V. Using thematic analysis in psychology. Qual Res Psychol 2006;3:77-101.

37. Applebaum AJ, Richardson MA, Brady SM, Brief DJ, Keane TM. Gender and other psychosocial factors as predictors of adherence to highly active antiretroviral therapy (HAART) in adults with comorbid HIV/AIDS, psychiatric and substance-related disorder. AIDS Behav 2009;13: 60-65.

38. Sebate E, ed. Adherence to Long-Term Therapies: Evidence for Action. Geneva: World Health Organization, 2003.

39. Nachega JB, Trotta MP, Nelson M, Ammassari A. Impact of metabolic complications on antiretroviral treatment adherence: Clinical and public health implications. Curr HIV/ AIDS Rep 2009;6:121-129.

40. Simoni JM, Amico KR, Smith L, Nelson K. Antiretroviral adherence interventions: translating research findings to the real world clinic. Curr HIV/AIDS Rep 2010;7:44-51.

41. Wolfe WR, Weiser SD, Bangsberg DR, et al. Effects of HIV-related stigma among an early sample of patients receiving antiretroviral therapy in Botswana. AIDS Care 2006;18:931-933.

42. Jewkes R, Dunkle K, Nduna M, et al. Factors associated with HIV sero-status in young rural South African women: Connections between intimate partner violence and HIV. Int J Epidemiol 2006;35:1461-1468.

43. Jewkes R, Levin J, Pen-Kekana L. Gender inequalities, intimate partner violence and HIV preventive practices: Findings of a South African cross-sectional study. Soc Sci Med 2003;56:125-134.

44. Okoror TA, Falade CO, Olorunlana A, Walker EM, Okareh OT. Exploring the cultural context of HIV stigma on antiretroviral therapy adherence among people living with
HIV/AIDS in southwest Nigeria. AIDS Patient Care STDS 2013;27:55-64.

45. Ware NC, Wyatt MA, Geng EH, et al. Toward an understanding of disengagement from HIV treatment and care in sub-Saharan Africa: A qualitative study. PLoS Med 2013;10:e1001369; discussion, e1001369.

46. Batavia AS, Balaji K, Houle E, et al. Adherence to antiretroviral therapy in patients participating in a graduated cost recovery program at an HIV care center in South India. AIDS Behav 2010;14:794-798.

47. Weiser S, Wolfe W, Bangsberg D, et al. Barriers to antiretroviral adherence for patients living with HIV infection and AIDS in Botswana. J Acquir Immune Defic Syndr 2003;34:281-288.

48. Abdool Karim SS, Naidoo K, Grobler A, et al. Timing of initiation of antiretroviral drugs during tuberculosis therapy. N Engl J Med 2010;362:697-706.

49. Abdool Karim SS, Naidoo K, Grobler A, et al. Integration of antiretroviral therapy with tuberculosis treatment. New Engl J Med 2011;365:1492-1501.

Address correspondence to: Dr. Francois van Loggerenberg

University of Oxford

The Global Health Network

Center for Tropical Medicine and Global Health

NDM Research Building Old Road Campus Oxford OX3 $7 F Z$ United Kingdom

E-mail: francois.vanloggerenberg@ndm.ox.ac.uk 